\title{
The association between cecal insertion time and colorectal neoplasm detection
}

\author{
Moon Hee Yang ${ }^{1}$, Juhee Cho ${ }^{2,3}$, Sanjay Rampal ${ }^{3,4}$, Eun Kyung Choi ${ }^{2}$, Yoon-Ho Choi ${ }^{1}$, Jun Haeng Lee ${ }^{5}$, \\ Young-Ho Kim ${ }^{5}$, Dong Kyung Chang ${ }^{5}$, Poong-Lyul Rhee ${ }^{5}$, Jae J Kim ${ }^{5}$, Eliseo Guallar ${ }^{3,6}$, Jong Chul Rhee \\ and Hee Jung Son ${ }^{1,5^{*}}$
}

\begin{abstract}
Background: Information on the impact of cecal insertion time on colorectal neoplasm detection is limited. Our objective was to determine the association between cecal insertion time and colorectal neoplasm detection rate in colonoscopy screening.

Methods: We performed a cross-sectional study of 12,679 consecutive subjects aged 40-79 years undergoing screening colonoscopy in routine health check-ups at the Center for Health Promotion of the Samsung Medical Center from December 2007 to June 2009. Fixed effects logistic regression conditioning on colonoscopist was used to eliminate confounding due to differences in technical ability and other characteristics across colonoscopists.

Results: The mean cecal insertion time was 5.9 (SD, 4.4 minutes). We identified 4,249 (33.5\%) participants with colorectal neoplasms, of whom 1,956 had small single adenomas $(<5 \mathrm{~mm}), 595$ had medium single adenomas (5-9 mm), and 1,699 had multiple adenomas or advanced colorectal neoplasms. The overall rates of colorectal neoplasm detection by quartiles of cecal insertion time were $36.8 \%, 33.4 \%, 32.7 \%$, and $31.0 \%$, respectively ( $p$ trend $<0.001$ ). The odds for small single colorectal adenoma detection was $16 \%$ lower (adjusted OR $0.84 ; 95 \% \mathrm{Cl} 0.71$ to 0.99 ) in the fourth compared to the first quartile of insertion time ( $p$ trend 0.005). Insertion time was not associated with the detection rate of single adenomas $\geq 5 \mathrm{~mm}$, multiple adenomas or advanced colorectal neoplasms.

Conclusion: Shorter insertion times were associated with increased rates of detection of small colorectal adenomas $<5 \mathrm{~mm}$. Cecal insertion time may be clinically relevant as missed small colorectal adenomas may progress to more advanced lesions.
\end{abstract}

Keywords: Cecal insertion time, Colorectal neoplasm, Screening colonoscopy

\section{Background}

Early detection and removal of colorectal adenomas by colonoscopy decreases the risk of colorectal cancer [1], and colonoscopy has become the standard for detection of colorectal neoplasms. However, the miss rate for colonoscopy is not negligible and up to $5 \%$ of new colorectal cancer cases had a colonoscopy in the previous 3-5 years [2-6]. Indeed, the adenoma detection rate is an independent predictor of the risk of interval colorectal cancer after

\footnotetext{
* Correspondence: hjls.son@samsung.com

${ }^{1}$ Center for Health Promotion, Samsung Medical Center, Sungkyunkwan University School of Medicine, Seoul, Korea

${ }^{5}$ Department of Internal Medicine, Samsung Medical Center, Sungkyunkwan University School of Medicine, 50 Irwon-dong, Gangnam-gu, Seoul 135-710, Korea

Full list of author information is available at the end of the article
}

screening colonoscopy [7], and measuring the adenoma detection rates of individual colonoscopists is a priority in quality improvement in colonosocopy units [8].

Higher adenoma detection rates are associated with more careful examination of the proximal sides of folds and flexures, with adequate distension, with cleaning of pools of fluid and dirty areas, and with time for scope withdrawal $[9,10]$. Taking adequate time during withdrawal is necessary for meticulous mucosal examination and several studies have demonstrated that colonoscopy withdrawal time is a major determinant of adenoma miss rates [10-13]. Shorter cecal insertion time in relation to withdrawal time was associated with higher adenoma detection rates in a previous small study [14]. However, more research is needed to determine the

\section{Biomed Central}


appropriateness of using insertion time as a quality indicator for colonoscopy. The aim of this study was to investigate the association between cecal insertion time and colorectal neoplasm detection in a large colonoscopy screening clinic.

\section{Methods \\ Study population}

We conducted a retrospective study of screening colonoscopies at the Center for Health Promotion of the Samsung Medical Center in Seoul, Korea, from December 2007 to June 2009. We restricted the study to male and female participants aged 40 to 79 years old as this is the recommended age range for screening colonoscopy in Korea. From a total of 14,370 consecutive colonoscopies performed at the Center for Health Promotion, we excluded 862 colonoscopies conducted in participants who were $<40$ or $\geq 80$ years of age, 526 colonoscopies performed for therapeutic (non-screening) purposes, 65 colonoscopies performed outside of the colonoscopy unit, 29 colonoscopies performed in participants with a history of colorectal cancer, inflammatory bowel disease or who had undergone colonic resection, and 113 colonoscopies with incomplete examination due to poor bowel preparation. In subjects who had colonoscopy multiple times during the study period ( $\mathrm{n}=98$ ), we chose the first colonoscopy results. Because some individuals met more than one exclusion criterion, the final sample size was 12,679 (7,975 men and 4,704 women). The study protocol was reviewed and approved by the institutional review board at Samsung Medical Center. The informed consent requirement was exempted by the Institutional Review Board because researchers only accessed retrospectively a de-identified database for analysis purposes.

\section{Study procedures}

Twenty three board-certified gastroenterologists performed the colonoscopies. Each colonoscopist had performed more than 500 examinations before the study period or before working at the Center for Health Promotion. Colonoscopies were performed after bowel preparation with $4 \mathrm{~L}$ polyethylene glycol solution (Colyte ${ }^{\circledR}$, Taejun, Seoul, Korea; Colyte ${ }^{\circledR}-$ F, Taejun, Seoul, Korea; Colonlyte $^{\circledR}$, Dreampharma, Seoul, Korea).

Cecal insertion time was defined as the time from insertion into the rectum to the time when the colonoscope tip passed to a point proximal to the ileocecal valve so that the base of cecum was visible. Withdrawal time was defined as the time taken for withdrawing the colonoscope tip from the base of cecum to across the anus. Cecal insertion and withdrawal times were recorded immediately after finishing the examination by the colonoscopist. Bowel preparation was assessed as excellent (no or nearly no fecal matter in the colon; small-to-moderate amounts of clear liquid present), good (small amounts of thin liquid fecal matter visible and easily suctioned, mainly distal to the splenic flexure), fair (moderate amounts of thick liquid to semisolid fecal matter visible and suctioned, including proximal to the splenic flexure; $>90 \%$ of the mucosa visible), or poor (large amounts of solid fecal matter present that preclude a satisfactory study; $<90 \%$ of the mucosa visible).

Colorectal neoplasms were further classified as small single non-advanced adenomas $<5 \mathrm{~mm}$, medium single non-advanced adenomas 5 to $<10 \mathrm{~mm}$,and multiple or advanced colorectal neoplasms (including multiple adenomas regardless of size and advanced colorectal neoplasms). Advanced adenoma was defined as a tubular adenoma with diameter $\geq 10 \mathrm{~mm}$, an adenoma with villous component, or an adenoma with high grade dysplasia. The size of each lesion was estimated using open biopsy forceps.

A health questionnaire and a detailed physical exam were routinely completed as part of the screening program. Height and weight were measured using an Inbody 720 machine (Biospace, Seoul, Korea). Body mass index (BMI) was calculated by dividing measured weight $(\mathrm{kg})$ by height squared $\left(\mathrm{m}^{2}\right)$. Waist circumference was measured at the midpoint between the inferior margin of the last rib and the superior iliac crest in a horizontal plane. Information on history of colorectal polyps, diabetes mellitus, hyperlipidemia, medication use including aspirin and non-steroidal anti-inflammatory drugs (NSAIDs), alcohol drinking and smoking and family history of colorectal cancer were collected using a selfadministered questionnaire before endoscopy.

\section{Statistical analysis}

Colorectal adenoma proportions were compared across the quartiles of cecal insertion time. Odds ratios (ORs) and $95 \%$ confidence intervals (CIs) for the presence of any colorectal neoplasm for the 3 highest quartiles of insertion time compared to the first quartile were calculated using fixed effects logistic regression conditioned on colonoscopists. These regression models account for data clustering by colonoscopists and eliminate confounding due to differences across colonoscopists. Separate logistic regression models were used for the different colorectal neoplasms. In multivariable models, we adjusted for age (40 to 49,50 to 59,60 to 69,70 to 79 years), sex, withdrawal time ( $<6$ minutes, $\geq 6$ minutes), bowel preparation (excellent, good, fair and poor), BMI $\left(<25, \geq 25 \mathrm{~kg} / \mathrm{m}^{2}\right)$, waist circumference $(<80,80$ to 89,90 to $99, \geq 100 \mathrm{~cm})$, family history of colorectal cancer, history of colorectal polyps, diabetes, hyperlipidemia, aspirin use, NSAIDs use, calcium use, alcohol drinking, and smoking (current, past, and never).

Responses to health questionnaire items coded as "unknown" and items not answered were considered as missing data. We used multiple imputations with chained 
equations to address missing data [15]. A total of $20 \mathrm{im}$ puted sets were created, each a result of 1000 iterations. Missing covariate patterns were individually explored and imputation equations refined. The residuals of the imputation regression models were graphically explored as a form of diagnostics. All analysis on imputed data accounted for the imputed nature of the datasets. Two sided $\mathrm{p}$-values $<0.05$ were considered statistically significant. Statistical analyses were performed using Stata version 12.0 (Stata Corp, Texas, USA).

\section{Results}

The mean (SD) age of study participants was 53.4(7.3) years and $62.9 \%$ of study participants were male (Table 1 ). The proportions of participants with a family history of colorectal cancer and with a personal history of colorectal polyps were $3.6 \%$ and $18.1 \%$, respectively. The mean (SD) cecal insertion time was 5.9 (4.4) minutes and the mean (SD) withdrawal time was 9.0 (3.9) minutes.

Their median age of the 23 colonoscopists was 34 years (interquartile range, 32 to 36). A majority of the colonoscopists were female (82.6\%). The median number of colonoscopies performed by each colonoscopist during the study period was 592 (interquartile range, 416 to 709 ). Before the study period, the 23 colonoscopists had performed a median of 1,400 colonoscopies (interquartile range, 1,200 to 1,900 ).

Colorectal neoplasms were detected in 4,249 participants (33.5\%; Table 2). The adenoma detection rates ranged from $23.7 \%$ to $48.3 \%$ across colonoscopists. The proportion of overall colorectal neoplasm detection by quartiles of cecal insertion time were 36.8\%, 33.4\%, $32.7 \%$, and $31.0 \%$ ( $\mathrm{p}$ trend $<0.001$ ). The detection rate of small single colorectal adenoma decreased with higher quartiles of insertion time: $17.7 \%, 15.1 \%, 14.9 \%$, and $13.9 \%$ ( $\mathrm{p}$ trend $<0.001$ ). The detection rate of multiple adenomas or advanced colorectal neoplasms also decreased with higher quartiles of insertion time: $14.4 \%$, $13.6 \%, 13.0 \%$, and $12.5 \%$ ( $\mathrm{P}$ trend 0.019 ). There was no evidence of an association between insertion time and the detection rates of single adenomas 5-9 $\mathrm{mm}$.

The inverse association between insertion time and small single colorectal adenoma persisted in multivariable analyses (Table 3). The odds for small single colorectal adenoma detection was 16\% lower (adjusted OR $0.84 ; 95 \%$ CI 0.71 to 0.99 ) in the fourth compared to the first quartile of insertion time ( $\mathrm{p}$ trend 0.005 ). Sensitivity analyses using complete case analyses $(\mathrm{N}=12,260)$ instead of multiple imputation for missing data showed similar results (Additional file 1: Table S1 and Additional file 2: Table S2). In analyses using insertion time as a continuous

Table 1 Characteristics of study participants $(N=12,679)$

\begin{tabular}{|c|c|c|c|}
\hline Characteristic & $\mathrm{N}$ available & $\%$ missing & Number (\%) or mean \pm SD \\
\hline Age, years & 12,679 & 0.0 & $53.4 \pm 7.3$ \\
\hline $40-49$ & & & $4,215(33.2)$ \\
\hline $50-59$ & & & $5,882(46.4)$ \\
\hline $60-69$ & & & $2,296(18.1)$ \\
\hline $70-79$ & & & $286(2.3)$ \\
\hline Male sex & 12,679 & 0.0 & $7,975(62.9)$ \\
\hline Body mass index, $\mathrm{kg} / \mathrm{m}^{2}$ & 12,091 & 4.6 & $24.2 \pm 2.8$ \\
\hline Waist circumference, $\mathrm{cm}$ & 10,803 & 14.8 & $84.1 \pm 8.8$ \\
\hline Family history of colorectal cancer & 12,679 & 0.0 & $458(3.6)$ \\
\hline History of colorectal polyp & 11,188 & 11.8 & $2,030(18.1)$ \\
\hline Diabetes mellitus & 11,237 & 11.4 & $975(8.7)$ \\
\hline Hyperlipidemia & 11,189 & 11.8 & $2,155(19.3)$ \\
\hline Aspirin use & 10,878 & 14.2 & $1,529(14.1)$ \\
\hline NSAIDs use & 10,878 & 14.2 & $321(3.0)$ \\
\hline Calcium use & 10,917 & 13.9 & $1,033(9.5)$ \\
\hline Currently consumes alcohol & 11,144 & 12.1 & $7,142(64.1)$ \\
\hline Current smoker & 11,406 & 10.0 & $2,150(18.8)$ \\
\hline \multicolumn{4}{|l|}{ Quality indicators of colonoscopy } \\
\hline Insertion time (minutes) & 12,260 & 3.3 & $5.9 \pm 4.4$ \\
\hline Withdrawal time (minutes) & 12,239 & 3.5 & $9.0 \pm 3.9$ \\
\hline Excellent/Good Bowel preparation & 11,805 & 6.9 & $8,086(68.5)$ \\
\hline
\end{tabular}


Table 2 Colorectal neoplasm detection by quartile of colonoscopy cecal insertion time $(\mathrm{N}=12,679)$

\begin{tabular}{|c|c|c|c|c|c|c|}
\hline & \multirow[t]{2}{*}{$\mathrm{N}(\%)$} & \multicolumn{5}{|c|}{ Quartiles of cecal insertion time, \% (SE\%) } \\
\hline & & $\begin{array}{c}\text { First } \\
(<3.1 \mathrm{~min})\end{array}$ & $\begin{array}{c}\text { Second } \\
(3.1-4.6 \mathrm{~min})\end{array}$ & $\begin{array}{c}\text { Third } \\
\text { (4.7-7.1 min) }\end{array}$ & $\begin{array}{c}\text { Fourth } \\
(\geq 7.2 \mathrm{~min})\end{array}$ & $\begin{array}{c}P \\
\text { value }\end{array}$ \\
\hline No colorectal lesion & $8,429(66.5)$ & $63.2(0.8)$ & $66.6(0.9)$ & $67.3(0.8)$ & $69.0(0.8)$ & \\
\hline Any colorectal lesion detection & $4,250(33.5)$ & $36.8(0.8)$ & $33.4(0.9)$ & $32.7(0.8)$ & $31.0(0.8)$ & $<0.001$ \\
\hline Small single adenoma, $<5 \mathrm{~mm}$ & $1,956(15.4)$ & $17.7(0.7)$ & $15.1(0.7)$ & $14.9(0.6)$ & $13.9(0.6)$ & $<0.001$ \\
\hline Medium single adenoma, 5-9 mm & $595(4.7)$ & $4.7(0.4)$ & $4.7(0.4)$ & $4.8(0.4)$ & $4.6(0.4)$ & 0.86 \\
\hline Multiple adenomas or advanced colorectal neoplasm & $1,699(13.4)$ & $14.4(0.6)$ & $13.6(0.6)$ & $13.0(0.6)$ & $12.5(0.6)$ & 0.019 \\
\hline
\end{tabular}

variable, the multivariable adjusted odds ratios (95\% CI) for colorectal neoplasm detection associated with a 5 minute increase in insertion time were 0.90 (95\% CI 0.84 to 0.97 ) for single adenomas $<5 \mathrm{~mm}, 1.05$ (0.94 to 1.16) for single adenomas 5 to $9 \mathrm{~mm}$ and 1.00 (0.93 to 1.07) for multiple adenomas or advanced colorectal neoplasms. In multivariable restricted cubic spline models, shorter cecal insertion times were also associated with higher detection rates of small $<5 \mathrm{~mm}$ single colorectal adenomas (Figure 1).

\section{Discussion and conclusions}

In this large study of routine screening colonoscopy practice, shorter cecal insertion times were associated with higher detection rates of adenomas, although the association was restricted to small adenomas. This association persisted after controlling for colonoscopist and after adjusting for a variety of patient and colonoscopy characteristics, including withdrawal time and bowel preparation. Since small adenomas comprise a large proportion of adenomas and missing them may increase the likelihood of colorectal cancers, our findings may be relevant from a clinical perspective.

Cecal insertion time is determined by multiple colonoscopist- and patient-related factors. Insertion time may reflect the colonoscopist's skill, concentration, or fatigue. Experienced colonoscopists inserting the scope to the cecum without forming colonic loops are likely to have shorter insertion times. Examining the colon after straightening allows for a more controlled pull back, with easier reinsertion to recheck difficult and potential blind areas [16]. Withdrawal through a large loop complicates control of the colonoscope and may result in skipping several inches of the colon without having been inspected at all [16]. A good insertion technique is thus essential for a good examination of the colonic mucosa. A fast and efficient insertion technique may also make it easier for the colonoscopist to keep his/her concentration during withdrawal. Insertion time may also be a marker for overall proficiency of the colonoscopist, as lower annual case volumes are associated with prolonged insertion times [17].

Table 3 Odds ratios for colorectal neoplasm detection by colonoscopy cecal insertion times $(\mathbf{N}=12,679)$

\begin{tabular}{|c|c|c|c|c|c|c|}
\hline & \multicolumn{6}{|c|}{ Quartiles of cecal insertion time, Odds Ratio $(95 \% \mathrm{Cl})$} \\
\hline & $\begin{array}{c}\text { First } \\
(<3.1 \mathrm{~min})\end{array}$ & $\begin{array}{c}\text { Second } \\
(3.1-4.6 \mathrm{~min})\end{array}$ & $\begin{array}{c}\text { Third } \\
(4.7-7.1 \mathrm{~min})\end{array}$ & $\begin{array}{c}\text { Fourth } \\
(\geq 7.2 \mathrm{~min})\end{array}$ & $\begin{array}{l}\text { Per 5-minute } \\
\text { increase }\end{array}$ & $\begin{array}{l}\mathrm{P}- \\
\text { trend }\end{array}$ \\
\hline \multicolumn{7}{|l|}{ Any colorectal neoplasms } \\
\hline Crude OR* $(95 \% \mathrm{Cl})$ & 1.00 (ref.) & $0.87(0.78,0.97)$ & $0.93(0.83,1.04)$ & $0.90(0.80,1.02)$ & $0.97(0.92,1.01)$ & 0.16 \\
\hline Adjusted OR** $(95 \% \mathrm{Cl})$ & 1.00 (ref.) & $0.95(0.85,1.06)$ & $1.01(0.89,1.13)$ & $0.94(0.83,1.07)$ & $0.96(0.91,1.01)$ & 0.13 \\
\hline \multicolumn{7}{|c|}{ Small single adenoma, $<5 \mathrm{~mm}$} \\
\hline Crude OR* $(95 \% \mathrm{Cl})$ & 1.00 (ref.) & $0.80(0.69,0.92)$ & $0.83(0.72,0.96)$ & $0.77(0.66,0.91)$ & $0.89(0.83,0.95)$ & 0.001 \\
\hline Adjusted OR** $(95 \% \mathrm{Cl})$ & 1.00 (ref.) & $0.87(0.75,1.00)$ & $0.91(0.78,1.06)$ & $0.84(0.71,0.99)$ & $0.90(0.84,0.97)$ & 0.005 \\
\hline \multicolumn{7}{|c|}{ Medium single adenoma, 5-9 mm } \\
\hline Crude OR* $(95 \% \mathrm{Cl})$ & 1.00 (ref.) & $1.00(0.78,1.28)$ & $1.10(0.86,1.41)$ & $1.08(0.83,1.41)$ & $1.05(0.94,1.16)$ & 0.37 \\
\hline Adjusted OR** $(95 \% \mathrm{Cl})$ & 1.00 (ref.) & $1.05(0.82,1.35)$ & $1.16(0.89,1.50)$ & $1.11(0.84,1.46)$ & $1.05(0.94,1.16)$ & 0.40 \\
\hline \multicolumn{7}{|c|}{ Multiple adenomas or advanced colorectal neoplasm } \\
\hline Crude OR* $(95 \% \mathrm{Cl})$ & 1.00 (ref.) & $0.93(0.80,1.08)$ & $0.99(0.85,1.16)$ & $1.02(0.86,1.20)$ & $1.02(0.96,1.09)$ & 0.52 \\
\hline Adjusted OR** $(95 \% \mathrm{Cl})$ & 1.00 (ref.) & $1.03(0.88,1.22)$ & $1.09(0.92,1.29)$ & $1.04(0.87,1.24)$ & $1.00(0.93,1.07)$ & 0.99 \\
\hline
\end{tabular}

* Crude OR conditions on colonoscopist.

** Adjusted OR adjusts for age, sex, body mass index, waist circumference, family history of colorectal cancer, history of colorectal polyp, diabetes mellitus, hyperlipidemia, aspirin medication, other NSAID medication, calcium supplementation, alcohol use, smoking history, colonoscopist, and bowel preparation. 


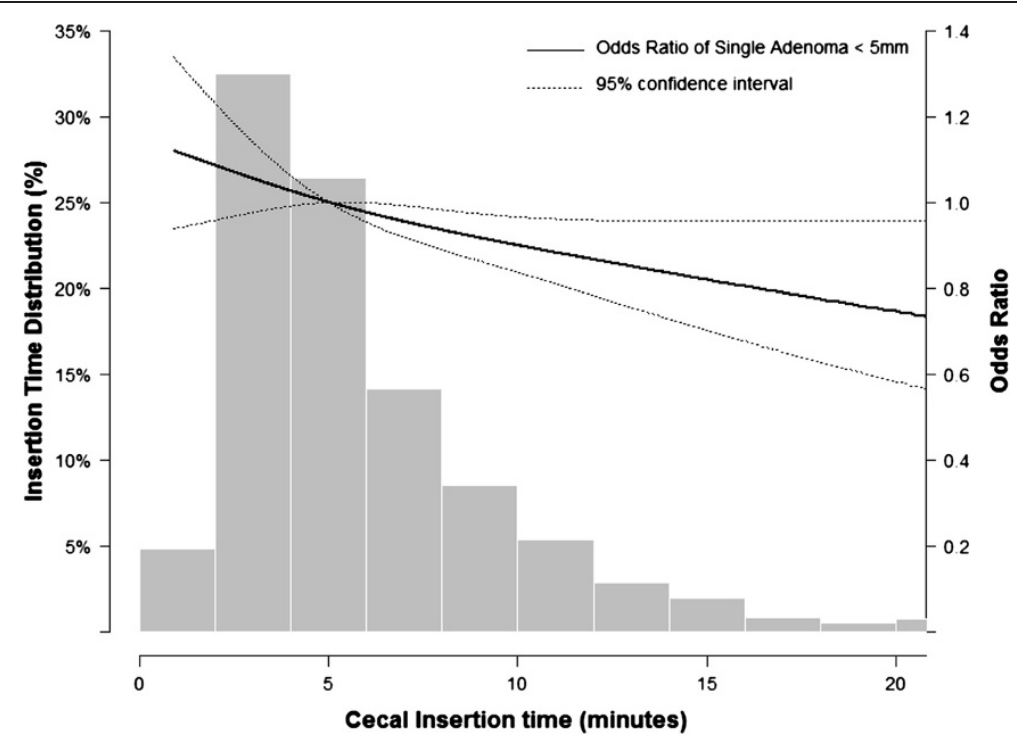

Figure 1 Prevalent odds ratio of single colorectal adenoma $<5 \mathrm{~mm}$. Adjusted relative prevalence odds ratios derived from spline logistic regression models using restricted cubic splines with knots at the 10th, 50th, and 90th percentiles of the distribution of the insertion time distribution. The reference value (odds ratio $=1$ ) was set at the 5 th minute of insertion time. Prevalent odds ratios were adjusted for age, sex, body mass index, waist circumference, family history of colorectal cancer, history of colorectal polyp, diabetes mellitus, hyperlipidemia, aspirin medication, other NSAID medication, calcium supplementation, alcohol use, smoking history, colonoscopist, and bowel preparation (see Table 3 for details).

We note, however, that our analyses conditioned on colonoscopist, so that variations in ability and other differences across colonoscopists cannot explain the inverse association that we observed between insertion time and small adenoma detection rate.

Prolonged insertion times also depend on patientrelated factors and may reflect a more difficult examination [18]. Prolonged cecal insertion time can be due to a redundant and tortuous colon, which may likely require prolonged withdrawal for a thorough inspection of the mucosa [14]. Older age, female sex, poor bowel preparation, smaller waist circumference, lower BMI, history of prior abdominal hysterectomy, and constipation have all been associated with increased cecal insertion times [17-20]. In our study, however, adjusting for a variety of procedure- and patient-related factors, including withdrawal time, did not materially affect the observed inverse association between insertion time and small adenoma detection. In a prior study, colonoscopists with mean cecal insertion to mean withdrawal time ratios $<1$ detected significantly more adenomas compared to colonoscopists with ratios $>1$ [14]. Stress from longer insertion times and scheduling pressure may influence the observed cecal intubation to withdrawal time relationship [14]. The higher miss rate in patients with longer cecal insertion times may thus be related to patient or colonic related factors, to endoscopists' fatigue from a difficult colonoscopy, or to stress from scheduling. Therefore, a longer insertion time may increase the likelihood that an endoscopist misses smaller adenomas in relatively difficult to visualize areas of the colon. We similarly found that cecal insertion time was negatively related to withdrawal time (results not shown). In our study, we used the insertion times of each colonoscopy instead of the mean insertion times of each colonoscopist and we controlled for colonoscopist to exclude that the observed association was due to other differences across colonoscopists. In another large study of primary care physician-performed colonoscopies with standby specialist support, mean insertion time was significantly longer in cases with polyps [21]. However, colonoscopies performed by primary care physicians with lower colonoscopy volumes and mean insertion times were much longer than those of experienced boardcertified gastroenterologists in other studies. A recent small study reported that insertion times $>5 \mathrm{~min}$ and withdrawal time $<10 \mathrm{~min}$ were associated with a higher miss rate [22], but this study was restricted to patients who underwent a second colonoscopy for polypectomy and its findings are not directly applicable to all patients undergoing screening colonoscopy.

While the clinical implications and optimal management of small polyps are controversial [23,24], several lines of evidence indicate that detection of small polyps may result in long-term benefits for patients [12,25]. In a close follow-up of 30 polyps during 2 years, the mean tubular adenoma growth rate was $0.58 \mathrm{~mm} /$ year and 3 tubular adenomas had growth rates $>2 \mathrm{~mm} /$ year [26]. No polyp regressed completely [26]. There is evidence that although 
the presence of cancer at the time of detection is uncommon, small adenomas may eventually develop into cancer [27]. Furthermore, small adenomas can be easily removed by hot or cold biopsy forceps, with lower risk of bleeding compared to polypectomy [27].

Our study had several strengths, including the large sample size, the use of unselected participants undergoing routine screening colonoscopy, and the availability of detailed covariate information which allowed us to consider in the analysis many patient- and procedure-related factors that affect the prevalence and detection rates of colorectal neoplasms. Also the use of fixed effects regression conditional on colonoscopist also eliminates confounding by potential differences across colonoscopists.

Several limitations to our study, however, also need to be considered in the interpretation of our findings. First, we could not determine whether polyps were detected or removed during insertion or withdrawal of the colonoscope. Removal of diminutive polyps during insertion, however, cannot explain an inverse association between shorter insertion times and adenoma detection rate. Second, we did not have data on the use of sedative or antispasmodic drugs during colonoscopy, which may influence cecal insertion time and adenoma detection rate. Third, our questionnaire captured history of colorectal polyps but not of colorectal adenomas, and some participants could not remember the results of previous colonoscopies. However, our findings were similar in the subgroup of study participants who reported a negative history of colorectal polyps. Fourth, we did not have information on compliance to preparation procedures. We could not assess the consistency of colonoscopists to adjudicate bowel preparation score criteria that may be subject to intra- and inter-observer variation. Fifth, we could not investigate practice-related factors, including number of colonoscopies performed per day and daily total procedure time that may influence colonoscopist performance. Sixth, polyp sizes were measured by eye using open biopsy forceps. Therefore, exact measurements of polyp size were not available and intra- and inter-observer variation may add to random variability of analyses by tumor size. Finally, as our study included endoscopists and patients from a single center, generalizability of these findings to other populations is uncertain.

In conclusion, we found an inverse association between cecal insertion time and the detection of small colorectal adenomas. Since missed colorectal adenomas may progress to more advanced lesions, our findings may be relevant from a clinical perspective. In our analysis, we controlled for the effects of individual endoscopists. As a consequence, our data suggest that endoscopists should pay close attention to the detection of adenomas in cases that need longer insertion times.

\section{Additional files}

Additional file 1: Table S1. Colorectal neoplasm detection by quartile of cecal colonoscopy insertion time (complete case analysis, $N=12,260$ ).

Additional file 2: Table S2. Odds ratios for colorectal neoplasm detection by cecal colonoscopy insertion times (Complete case analysis).

Competing interest

The authors declare that they have no competing interest.

\section{Authors' contributions}

Conception and design: MHY, JC, JHL, HJS, EG. Acquisition of data: MHY, EKC, Y-HC, JHL, Y-HK, DKC, P-LR, JJK, JCR. Analysis and interpretation of data: MHY, JC, SR, EG, HJS. Manuscript writing: MHY, JC, SR, EG. Critical revision of manuscript: EG, JHL, Y-HK, DKC, P-LR, JJK, JCR, Y-HC, EKC, HJS. Study supervision: EG, HJS. Final approval of manuscript: EG, HJS, MHY, JC, SR, EKC, Y-HC, JHL, Y-HK, DKC, $\mathrm{P}-\mathrm{LR}$, JJK, JCR. All authors read and approved the final manuscript.

\section{Author details}

${ }^{1}$ Center for Health Promotion, Samsung Medical Center, Sungkyunkwan University School of Medicine, Seoul, Korea. ${ }^{2}$ Cancer Education Center Samsung Medical Center, Sungkyunkwan University School of Medicine, Seoul, Korea. ${ }^{3}$ Department of Epidemiology, Johns Hopkins University Bloomberg School of Public Health, Baltimore, USA. ${ }^{4}$ Julius Centre University of Malaya, Department of Social and Preventive Medicine, Faculty of Medicine, University of Malaya, Kuala Lumpur, Malaysia. ${ }^{5}$ Department of Internal Medicine, Samsung Medical Center, Sungkyunkwan University School of Medicine, 50 Irwon-dong, Gangnam-gu, Seoul 135-710, Korea. ${ }^{6}$ Department of Medicine and Welch Center for Prevention, Epidemiology, and Clinical Research, Johns Hopkins Medical Institutions, Baltimore, USA.

Received: 7 December 2012 Accepted: 30 July 2013

Published: 6 August 2013

\section{References}

1. Citarda F, Tomaselli G, Capocaccia R, Barcherini S, Crespi M: Efficacy in standard clinical practice of colonoscopic polypectomy in reducing colorectal cancer incidence. Gut 2001, 48:812-815.

2. Bretagne JF, Ponchon $\mathrm{T}$ : Do we need to embrace adenoma detection rate as the main quality control parameter during colonoscopy? Endoscopy 2008, 40:523-528.

3. Bressler B, Paszat LF, Vinden C, Li C, He J, Rabeneck L: Colonoscopic miss rates for right-sided colon cancer: a population-based analysis. Gastroenterology 2004, 127:452-456.

4. Bressler B, Paszat LF, Chen Z, Rothwell DM, Vinden C, Rabeneck L: Rates of new or missed colorectal cancers after colonoscopy and their risk factors: a population-based analysis. Gastroenterology 2007, 132:96-102.

5. Farrar WD, Sawhney MS, Nelson DB, Lederle FA, Bond JH: Colorectal cancers found after a complete colonoscopy. Clin Gastroenterol Hepatol 2006, 4:1259-1264.

6. Van Rijn JC, Reitsma JB, Stoker J, Bossuyt PM, Van Deventer SJ, Dekker E: Polyp miss rate determined by tandem colonoscopy: a systematic review. Am J Gastroenterol 2006, 101:343-350.

7. Kaminski MF, Regula J, Kraszewska E, Polkowski M, Wojciechowska U, Didkowska J, Zwierko M, Rupinski M, Nowacki MP, Butruk E: Quality indicators for colonoscopy and the risk of interval cancer. $N$ Engl J Med 2010, 362:1795-1803.

8. Rex DK, Petrini JL, Baron TH, Chak A, Cohen J, Deal SE, Hoffman B, Jacobson BC, Mergener K, Petersen BT, Safdi MA, Faigel DO, Pike IM: Quality indicators for colonoscopy. Am J Gastroenterol 2006, 101:873-885.

9. Rex DK: Maximizing detection of adenomas and cancers during colonoscopy. Am J Gastroenterol 2006, 101:2866-2877.

10. Rex DK: Colonoscopic withdrawal technique is associated with adenoma miss rates. Gastrointest Endosc 2000, 51:33-36.

11. Sanchez W, Harewood GC, Petersen BT: Evaluation of polyp detection in relation to procedure time of screening or surveillance colonoscopy. Am J Gastroenterol 2004, 99:1941-1945.

12. Barclay RL, Vicari JJ, Doughty AS, Johanson JF, Greenlaw RL: Colonoscopic withdrawal times and adenoma detection during screening colonoscopy. N Engl J Med 2006, 355:2533-2541. 
13. Millan MS, Gross P, Manilich E, Church JM: Adenoma detection rate: the real indicator of quality in colonoscopy. Dis Colon Rectum 2008, 51:1217-1220.

14. Benson ME, Reichelderfer M, Said A, Gaumnitz EA, Pfau PR: Variation in colonoscopic technique and adenoma detection rates at an academic gastroenterology unit. Dig Dis Sci 2010, 55:166-171.

15. White IR, Royston P, Wood AM: Multiple imputation using chained equations: issues and guidance for practice. Stat Med 2011, 30:377-399.

16. Church J: Adenoma detection rate and the quality of colonoscopy: the sword has two edges. Dis Colon Rectum 2008, 51:520-523.

17. Bernstein C, Thorn M, Monsees K, Spell R, O'Connor JB: A prospective study of factors that determine cecal intubation time at colonoscopy. Gastrointest Endosc 2005, 61:72-75.

18. Hsieh YH, Kuo CS, Tseng KC, Lin HJ: Factors that predict cecal insertion time during sedated colonoscopy: the role of waist circumference. J Gastroenterol Hepatol 2008, 23:215-217.

19. Cirocco WC, Rusin LC: Factors that predict incomplete colonoscopy. Dis Colon Rectum 1995, 38:964-968.

20. Kim WH, Cho YJ, Park JY, Min PK, Kang JK, Park IS: Factors affecting insertion time and patient discomfort during colonoscopy. Gastrointest Endosc 2000, 52:600-605.

21. Xirasagar S, Hurley TG, Sros L, Hebert JR: Quality and safety of screening colonoscopies performed by primary care physicians with standby specialist support. Med Care 2010, 48:703-709.

22. Kim JH, Kim YS, Cheon JH, Lee SK, Kim Tl, Myoung S, Kim WH: Influence of the insertion time and number of polyps on miss rate in colonoscopy. Scand I Gastroenterol 2011, 46:634-639.

23. Lenhart DK, Zalis ME: Debate: diminutive polyps noted at CT colonography need not be reported. Gastrointest Endosc Clin N Am 2010, 20:227-237

24. Lieberman D: Debate: small $(6-9 \mathrm{~mm})$ and diminutive $(1-5 \mathrm{~mm})$ polyps noted on CTC: how should they be managed? Gastrointest Endosc Clin N Am 2010, 20:239-243.

25. Church JM: Clinical significance of small colorectal polyps. Dis Colon Rectum 2004, 47:481-485.

26. Bersentes K, Fennerty MB, Sampliner RE, Garewal HS: Lack of spontaneous regression of tubular adenomas in two years of follow-up. Am J Gastroenterol 1997, 92:1117-1120.

27. Lewis B: The only good polyp. Am J Gastroenterol 1995, 90:1-2.

doi:10.1186/1471-230X-13-124

Cite this article as: Yang et al:: The association between cecal insertion time and colorectal neoplasm detection. BMC Gastroenterology 2013 13:124

\section{Submit your next manuscript to BioMed Central and take full advantage of:}

- Convenient online submission

- Thorough peer review

- No space constraints or color figure charges

- Immediate publication on acceptance

- Inclusion in PubMed, CAS, Scopus and Google Scholar

- Research which is freely available for redistribution 